\title{
Diagnostic Errors in the Neonatal Intensive Care Unit: A Case Series
}

\author{
Grant J. Shafer, MD ${ }^{1,2}$ Gautham Suresh, $\mathrm{MD}^{1,2}$ \\ ${ }^{1}$ Division of Neonatal-Perinatal Medicine, Department of Pediatrics, \\ Baylor College of Medicine, Houston, Texas \\ 2 Department of Neonatology, Texas Children's Hospital, \\ Houston, Texas \\ Am J Perinatol Rep 2018;8:e379-e383.
}

Address for correspondence Grant J. Shafer, MD, Department of Neonatology, Texas Children's Hospital, 6621 Fannin Street, Suite W6014, Houston, TX, 77030 (e-mail: gshafer@bcm.edu).

\author{
Abstract \\ Keywords \\ - diagnostic errors \\ - case series \\ - neonatal intensive \\ care unit \\ - neonatology
}

Diagnostic errors remain understudied in neonatal intensive care units (NICUs). The few available studies are primarily autopsy-based, and do not evaluate diagnostic errors that did not result in the patient's death. This case series presents 10 examples of nonlethal diagnostic errors in the NICU-classified according to the component of the diagnostic process which led to the error. These cases demonstrate the presence of diagnostic error in the NICU and highlight the need for further research on this important topic.
A diagnostic error is defined as "a failure to establish an accurate and timely explanation for a patient's health problem." Such errors cause significant harm to patients, and were identified as a priority by the National Academy of Medicine in its 2015 report, Improving Diagnosis in Health Care. However, diagnostic errors remain largely unappreciated within the quality and patient safety movement in health care. ${ }^{2,3}$ In particular, diagnostic errors within a neonatal intensive care unit (NICU) are understudied. ${ }^{2}$ NICU patients are fragile and often require multiple invasive interventions in a fast-paced, complex environment. As a result, they are at high risk of diagnostic errors that result in significant short-term and long-term health consequences. ${ }^{4}$ To date, however, the few studies on diagnostic errors in the NICU have been autopsy-based evaluations that did not assess nonlethal diagnostic errors. ${ }^{4,5}$

In this case series, we present 10 examples of diagnostic errors which occurred in the NICU at this institution. These cases were identified by us during the course of our clinical work over a period of 9 months. We classify the errors by the component of the diagnostic process which led to the error in diagnosis: missed physical exam findings, misinterpreted vital signs, incorrectly interpreted radiographic imaging, delayed laboratory result interpretation, incorrectly interpreted echocardiographic imaging, inadequate team communication, and no fault. These cases serve as prototypical examples of diagnostic errors in the NICU, and highlight the need for further research in this field.

\section{Cases}

\section{Missed Physical Examination Findings}

\section{Case 1}

A 31-week gestation infant was born by spontaneous vaginal delivery (SVD) after preterm labor and prolonged (1 week) preterm premature rupture of membranes. The mother had received regular prenatal care, had negative results on prenatal tests for infections, and denied any history of herpes simplex virus (HSV) infection. On admission, the eyes were clear without discharge, with a bilateral red reflex. The patient had an uncomplicated NICU course until 5 weeks of age, when the infant developed eye redness and eyelid swelling of the right eye. Examination by an ophthalmologist revealed a geographic ulcer with central large epithelial defect that was suggestive of HSV keratitis. No eye exam had been documented in the daily progress notes in the week prior to the ophthalmology review. A viral culture of the eye discharge yielded HSV type 2. Blood and cerebral spinal fluid (CSF) cultures were negative for HSV and other infections. The patient received a 21-day treatment course of intravenous acyclovir as well as topical ganciclovir, then was switched to PO acyclovir only for suppressive therapy. received

August 14, 2018

accepted after revision

November 3, 2018
DOI https://doi.org/

10.1055/s-0038-1676619. ISSN 2157-6998.
Copyright $\odot 2018$ by Thieme Medical Publishers, Inc., 333 Seventh Avenue, New York, NY 10001, USA. Tel: +1(212) 584-4662.
License terms

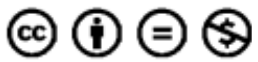


The patient has now been discharged from the NICU, and remains on $\mathrm{PO}$ acyclovir at home, which is being managed by the infectious disease outpatient team. The plan is for 12 months of suppressive therapy with PO acyclovir. The patient is also being followed in the ophthalmology clinic with slow improvement of the scarring from the infection noted in the most recent clinic notes. The patient's long-term vision prognosis remains unclear.

\section{Case 2}

A 40-week gestation infant was born by SVD after an uncomplicated pregnancy, then admitted to the well-baby nursery, where the admitting pediatrician documented a normal exam -including a patent and normally placed anal opening. Enteral feedings were then started. At 10 hours of life, the patient developed emesis and a distended abdomen. On reassessment, the infant was noted to have an imperforate anus. After transfer to the NICU a membrane covering a perineal fistula was excised by the pediatric surgery team at the bedside. Subsequently the patient fed well and passed stools normally.

\section{Misinterpreted Vital Sign Findings}

\section{Case 3}

A 37-week gestation infant was born by scheduled cesarean section (C-section) delivery. Prenatally multiple fetal congenital anomalies were identified, and fetal magnetic resonance imaging (MRI) demonstrated bilateral cerebral ventriculomegaly with mass effect on the cerebellum, caudal regression spectrum, kyphosis, and scoliosis. A fetal echocardiogram (echo) revealed a ventricular septal defect (VSD) and biventricular hypertrophy. The cardiologist recommended an echo after delivery. On admission to the NICU, the systolic blood pressure in the upper limb was documented to be higher than that in the lower limb by $30 \mathrm{~mm} \mathrm{Hg}$. This result-a 'red flag' for aortic coarctation-was recorded in the electronic medical record, but was not flagged as abnormal or communicated by the bedside nurse to the neonatology clinician. Neither the neonatologist nor the cardiologist recognized this concerning vital sign during their initial review of the chart, and this abnormal result is not mentioned in any of the notes from the day of admission. As the patient was otherwise hemodynamically stable after being intubated, a postnatal echo was deferred until the following day, while imaging of the head and kidneys was performed that night. Aortic coarctation was suspected on an echo at 22 hours of life, and confirmed on computed tomography (CT) angiography scan. The patient received a prostaglandin E1 infusion to maintain systemic perfusion for several days, until the parents elected to redirect care due to the multiple other congenital anomalies not consistent with long-term survival.

\section{Incorrectly Interpreted Radiographic Imaging and Delayed Laboratory Result Interpretation}

\section{Case 4}

A 34-week gestation infant was born by C-section, then admitted to the NICU for prematurity and hypoglycemia. An umbilical venous catheter (UVC) could not be inserted, so a peripherally inserted central catheter (PICC) was inserted into the right femoral vein. Afterward, a radiograph of the chest and abdomen was obtained to confirm correct positioning of the PICC. The radiology report described "a bubbly pattern in the descending colon," which was suggestive of pneumatosis. The pneumatosis was an incidental finding separate from the PICC insertion procedure, the placement of which did not have any influence on the development of the pneumatosis. There is a system in place at this institution by which a radiologist directly communicates concerning findings on imaging to the bedside clinician, but in this case it was not utilized by the radiologist who read the radiograph, and the neonatologist remained unaware of pneumatosis or the comments on the radiology report. As the radiograph had been obtained for PICC placement, and the patient appeared clinically well, the neonatologist initiated enteral feeds. At 14 hours of life, a blood culture grew gram-negative rods (later identified as Escherichia coli). This was also not recognized by the on-call neonatologist for 6 hours. During this period, the enteral feeds were continued, and no repeat blood cultures were sent until 20 hours of life, when the patient developed bloody stools. A repeat radiograph at that time demonstrated diffuse pneumatosis consistent with necrotizing enterocolitis. Feeds were then discontinued, and a full sepsis evaluation including a lumbar puncture for CSF culture was performed. The infant was treated with antibiotics, required multiple surgical interventions for colonic stricture, and experienced long-term feeding difficulties.

\section{Incorrectly Interpreted Radiographic Imaging}

\section{Case 5}

A 24-week gestation infant was born by SVD after preterm labor. At 2 weeks of life, the patient developed abdominal distension. An abdominal radiograph was obtained to evaluate for an etiology of the abdominal distension such as pneumoperitoneum or pneumatosis. The radiograph demonstrated diffuse gaseous distension of the colon and small bowel, and also noted a right pleural effusion. However, as this was an abdominal radiograph, the lungs were not completely evaluated in this study. The radiology report indicates that the finding of the pleural effusion was communicated to "the physician taking care of the patient," however, this was not noted in any of the documentation in the electronic medical record. Additionally, a dedicated follow-up chest radiograph was not obtained to fully evaluate the lungs. Instead the neonatology team focused on the abdominal distension, as the patient became critically ill with significant metabolic acidosis and decreased urine output concerning for an acutely evolving intraabdominal pathology. The following day, however, the patient had an acute respiratory decompensation with prolonged desaturations not responsive to manual positive pressure breaths or increases in fraction of inspired oxygen $\left(\mathrm{fiO}_{2}\right)$ on the ventilator. A chest radiograph obtained at that time demonstrated a large pleural effusion with leftward mediastinal shift, which required an emergent needle thoracentesis and chest tube placement. The patient was found to have coagulase-negative Staphylococcus sepsis, which caused a 
septic ileus that led to the initial abdominal distension. The pleural effusion was a separate clinical event that temporally coincided with the abdominal distension, but was not recognized by the medical team as they prioritized evaluation for an intra-abdominal pathology, and initially ascribed the respiratory distress as secondary to a 'competitive abdomen' given the abdominal distension.

\section{Case 6}

A 40-week gestation infant was born by SVD. The mother had regular prenatal care with unremarkable prenatal imaging. After delivery, the patient was noted to have sustained desaturations, so the infant was transferred to NICU for further evaluation. A chest radiograph on admission demonstrated 13 ribs, which was not noted on the radiologist's report or recognized by the on-call neonatologist. The following day, the NICU team noted the presence of an additional rib on the radiograph as well as concern for cardiomegaly. At this time, the patient continued to have desaturations and developed clinical signs and symptoms of respiratory distress (retractions and tachypnea) including stridor. Further work-up was undertaken at that time of multiple organ systems. Follow-up chest radiographs confirmed the presence of 13 ribs. An echo demonstrated significant outflow tract dilation of both the aortic root and pulmonary artery in addition to large atrial septal defect and VSD. Bedside, flexible nasopharyngoscopy by a pediatric otolaryngologist demonstrated paralysis of the left vocal cord, which was the cause of the stridor. Renal and head ultrasounds (USs) were obtained and both were normal. This cardiac abnormality was concerning for an underlying genetic etiology, so a whole exome sequencing genetic test was performed at the request of the genetics team. The testing identified a single missense variant (c.829T > A; p.W277R) in the TGFBR1 gene consistent with Loeys-Dietz type 1. The parents were counseled extensively on the diagnosis, then elected to proceed with cardiac repair. The patient has since been discharged from the NICU, and is being followed outpatient in the Cardiology, Genetics and Complex Care clinics. While the diagnostic error in this case did not markedly impact the patient's clinical status, we included it in this case series to highlight a diagnostic error which did not markedly impact the final outcome, but it demonstrates potential for improvement in the diagnostic process for the future.

\section{Case 7}

A 39-week gestation infant was born by scheduled C-section after a prenatal diagnosis of severe left-sided congenital diaphragmatic hernia $(\mathrm{CDH})$. The patient was treated with extracorporeal membrane oxygenation (ECMO) for 16 days after delivery and underwent surgical repair of the $\mathrm{CDH}$ while on ECMO. After ECMO, the patient required long-term ventilator support in the NICU as well as tracheostomy and gastrostomytube (g-tube) placement. The patient was on prolonged diuretic therapy due to chronic lung disease. At 5 months of age, the patient had persistent feeding intolerance, so a CT abdomen was obtained to evaluate for reherniation of the $\mathrm{CDH}$. Incidentally, the $\mathrm{CT}$ report noted a $5-\mathrm{mm}$ nonobstructing calculus in the lower pole of the left kidney. This was not followed up on or noted in the patient's chart by the neonatology team. At 7 months of age, the patient developed persistent hematuria. A renal US was obtained to evaluate for a cause of the hematuria and noted the calculus-which remained nonobstructive, but had grown in size. The neonatology team then became aware of the prior CT report of the calculus-the source of the persistent hematuria. The feeding difficulties slowly improved, and the patient was eventually able to tolerate full enteral feeds. As the renal calculus was not obstructive, the neonatology clinicians in consultation with the pediatric urology service elected to follow with serial imaging. The calculus has remained stable in size and no evidence of obstruction on follow-up USs. The hematuria has since resolved. If the calculus becomes obstructive or if the patient develops a urinary tract infection or urosepsis in the future, then the plan is for urologic intervention at that time.

\section{Incorrectly Interpreted Echocardiographic Imaging}

\section{Case 8}

A 34-week gestation infant was born by an ex utero intrapartum treatment (EXIT) procedure for severe left-sided CDH after inutero placement of an airway balloon by a fetal surgeon to improve lung growth. Following a prenatal diagnosis of $\mathrm{CDH}$ and in-utero placement of an airway balloon by a fetal surgeon, the mother developed preterm labor, so the infant was delivered at 34 weeks of gestation by the EXIT procedure. A prenatal echo had demonstrated normal cardiac anatomy. A postnatal echo on the day of birth identified a pulmonary vein on the right side returning to the left atrium, but did not visualize a pulmonary vein on the left side. This was not followed up by the neonatologist or cardiologist. The patient was treated with ECMO and surgical repair of the $\mathrm{CDH}$, followed by a prolonged NICU stay requiring long-term ventilator support. Over the course of 3 months, nine limited echos were performed to evaluate for pulmonary hypertension, but the full cardiac anatomy was not assessed on these studies. Several months later, a CT heart was obtained to evaluate for another medical issue, which incidentally noted partial anomalous pulmonary venous return (PAPVR) of the left lingular and lower pulmonary veins, which formed a confluent channel draining to the left innominate vein. This was confirmed by a repeat full anatomic echo. After further discussion with the cardiology and cardiothoracic surgery clinicians, it was determined that while this finding had been missed on previous studies, surgical intervention was not warranted at that time. The patient remained in the NICU for several months, and required tracheostomy placement due to inability to safely wean from the ventilator. There were no additional concerns from a cardiac standpoint during admission. The patient has now been discharged from the NICU, and is being followed in the cardiology clinic. The plan from a cardiac perspective is to allow the patient to continue to grow, then for surgical repair of the PAPVR at a yet to be determined date in the future.

\section{Inadequate Team Communication}

\section{Case 9}

A 31-week gestation infant was born by C-section due to poor fetal biophysical profile. A prenatal US had noted that the 
stomach was not visible. A subsequent US did not visualize the stomach or other portions of the gastrointestinal (GI) tract, a finding suggestive of tracheoesophageal fistula (TEF) or other GI tract abnormality. The obstetric team communicated the concern regarding the GI tract to the neonatologist attending the delivery, but these concerns were not relayed to the admitting neonatologist. No diagnostic testing was performed for GI anomalies. An abdominal radiograph performed to evaluate placement of an UVC noted an incidental finding of a rounded air collection in the upper mediastinum. An orogastric tube (OGT) was placed, but could not be advanced, and a radiograph demonstrated the OGT in the upper mediastinum with air distally in the GI tract, a finding suggestive of esophageal atresia with a TEF. This was confirmed on subsequent radiograph, in which the OGT was curled in the upper esophagus, and could not be advanced distally due to esophageal atresia. The decision was made to proceed to the operating room for surgical repair, and upon thoracotomy by the pediatric surgery team, and a type-C TEF was found and subsequently repaired. The patient had an uneventful recovery postoperatively, however, did require g-tube placement for enteral feeds due to inadequate oral feeding skills. The patient was discharged after several months in the NICU breathing room air and on full enteral feeds via the g-tube. Since discharge, the patient has required esophageal dilation by pediatric surgery.

\section{No Fault}

\section{Case 10}

A 34-week gestation infant was born by stat C-section due loss of fetal heart tones during routine fetal monitoring while the mother was at a prenatal appointment. During the pregnancy, the patient was diagnosed with multiple congenital anomalies, and the mother had undergone a fetal MRI, which demonstrated atresia of the jejunum with perforation, formation of meconium pseudocyst, lower urinary tract obstruction, arthrogryposis, and bilateral clubfeet. Prenatal genetic testing showed a chromosome 6 triplication. The patient was treated with intubation for respiratory distress, mechanical ventilation, a laparotomy to excise the jejunal atresia and a meconium pseudocyst, and creation of a jejunostomy. Postoperatively, the patient required prolonged mechanical ventilation, vasoactive support, and high-dose steroid administration. Genetic testing confirmed a chromosome 6 triplication. The patient had persistent hyperglycemia, which was initially ascribed to high-dose steroid administration. An insulin drip was initiated, but the patient's blood glucoses remained persistently elevated. An endocrinology consult and a literature review yielded the information that neonatal diabetes can be associated with chromosome 6 triplication. The patient was ultimately diagnosed with neonatal diabetes secondary to the chromosomal abnormality, and not due to high-dose steroid requirement as initially thought. Due to the extremely rare nature of this chromosome 6 triplication, the association with neonatal diabetes would not generally be considered in the typical differential diagnosis for neonatal hyperglycemia.

\section{Discussion}

This case series, the first of its kind to describe nonlethal diagnostic errors in the NICU, includes 10 cases categorized as cognitive, systems-based, or no-fault diagnostic errors. ${ }^{6}$ The majority were cognitive in origin (see - Table 1). We included an example of a no-fault diagnostic error-defined as a highly atypical presentation which is undetectable in

Table 1 Initial presentation, missed diagnosis, main disease category, and etiology of the diagnostic error for the examples of diagnostic error presented in this case series

\begin{tabular}{|c|c|c|c|c|}
\hline $\begin{array}{l}\text { Case } \\
(n=10)\end{array}$ & Initial presentation & Missed diagnosis & Main disease category & $\begin{array}{l}\text { Etiology of the } \\
\text { diagnostic error }\end{array}$ \\
\hline 1 & Premature delivery at $31 \mathrm{wk}$ & HSV keratitis & $\begin{array}{l}\text { Infectious disease and } \\
\text { ophthalmologic }\end{array}$ & Cognitive \\
\hline 2 & Term delivery & Imperforate anus & Gastrointestinal & Cognitive \\
\hline 3 & Multiple congenital anomalies & Coarctation of the aorta & Cardiovascular & Cognitive \\
\hline 4 & $\begin{array}{l}\text { Premature delivery at } 34 \text { wk } \\
\text { after maternal PPROM }\end{array}$ & NEC and E. Coli bacteremia & $\begin{array}{l}\text { Gastrointestinal and } \\
\text { infectious disease }\end{array}$ & $\begin{array}{l}\text { Cognitive and } \\
\text { systems-based }\end{array}$ \\
\hline 5 & $\begin{array}{l}\text { Premature delivery at } 24 \text { wk } \\
\text { with abdominal distension }\end{array}$ & Pleural effusion & Respiratory & $\begin{array}{l}\text { Cognitive and } \\
\text { systems-based }\end{array}$ \\
\hline 6 & Term delivery with desaturations & 13 ribs & Orthopedic & Cognitive \\
\hline 7 & $\mathrm{CDH}$ with hematuria & Renal calculus & Renal & Cognitive \\
\hline 8 & $\mathrm{CDH}$ with ventilator dependence & PAPVR & Cardiovascular & Cognitive \\
\hline 9 & Premature delivery at $31 \mathrm{wk}$ & TEF & Gastrointestinal & Systems-based \\
\hline 10 & $\begin{array}{l}\text { Persistent hyperglycemia in a } \\
\text { critically-ill neonate with } \\
\text { multiple congenital abnormalities }\end{array}$ & $\begin{array}{l}\text { Neonatal diabetes related to } \\
\text { chromosome } 6 \text { duplication }\end{array}$ & Endocrine & No fault \\
\hline
\end{tabular}

Abbreviations: $\mathrm{CDH}$, congenital diaphragmatic hernia; E. coli, Escherichia coli; HSV, herpes simplex virus; NEC, necrotizing enterocolitis; PAPVR, partial anomalous pulmonary venous return; PPROM, preterm premature rupture of membranes; TEF, tracheoesophageal fistula. 
spite of adequate diagnostic work-up ${ }^{6}$-to illustrate a diagnosis which would remain indiscernible despite a reasonable diagnostic evaluation, and thus would most likely not be amenable to interventions directed at improving diagnostic errors. Some of the cases had multiple diagnostic errors or involved multiple medical teams, which demonstrates the often multifactorial nature of diagnostic errors in a complicated environment such as the NICU. The outcome of these diagnostic errors varied as well-from no obvious harm, as in the patient with the missed radiologic finding of 13 ribs, to life-threatening, as in the patient with the missed pleural effusion requiring emergent needle thoracentesis. Fortunately, no patient died from diagnostic errors in this case series. We identified these diagnostic errors during the course of our clinical work in our NICU without any attempt to systematically perform surveillance for such errors, and described these cases to raise awareness of this problem in neonatology, and to highlight the need to systematically study these errors, which have been thus far relatively neglected in the patient safety movement. The chief limitation of our study is our inability to perform an in-depth analysis of the causal and contributory factors (particularly the cognitive processes involved) to the diagnostic errors we described. Therefore the categorization of these errors is solely based on the information available to us on chart review. In conclusion, a wide range of diagnostic errors may occur in NICU patients, and further research should address methods to identify, measure, and classify these errors and their impact. We are currently conducting a formal study to determine the incidence, types, and impact of diagnostic errors in the NICU, and we are using the cases described in this manuscript as prototypical cases to develop and refine our detection instrument, a modification of the Safer Dx instrument. $^{7}$

\section{Financial Disclosure}

The authors have no financial relationships relevant to this article to disclose.

\section{Funding Source}

No funding was secured for this manuscript.

Conflicts of Interest

The authors have no conflicts of interest relevant to this article to disclose.

\section{References}

1 Committee on Diagnostic Error in Health Care; Board on Health Care Services; Institute of Medicine et al. Improving Diagnosis in Health Care. Washington, DC: National Academies Press; 2015

2 Graber ML. The incidence of diagnostic error in medicine. BMJ Qual Saf 2013;22(Suppl 2):ii21-ii27

3 Graber M. Diagnostic errors in medicine: a case of neglect. Jt Comm J Qual Patient Saf 2005;31(02):106-113

4 Custer JW, Winters BD, Goode V, et al. Diagnostic errors in the pediatric and neonatal ICU: a systematic review. Pediatr Crit Care Med 2015;16(01):29-36

5 Widmann R, Caduff R, Giudici L, et al. Value of postmortem studies in deceased neonatal and pediatric intensive care unit patients. Virchows Arch 2017;470(02):217-223

6 Graber M, Gordon R, Franklin N. Reducing diagnostic errors in medicine: what's the goal? Acad Med 2002;77(10):981-992

7 Singh H, Sittig DF. Advancing the science of measurement of diagnostic errors in healthcare: the Safer Dx framework. BMJ Qual Saf 2015;24(02):103-110 